\title{
Modelos de Adopción de Tecnologías de la Información y Cloud Computing en las Organizaciones
}

\author{
Pedro Palos-Sanchez ${ }^{(1)}$, Ana Reyes-Menendez ${ }^{(2)}$ y Jose Ramon Saura(2)* \\ (1) Facultad de Ciencias Económicas y Empresariales, Dpto. de Administración de Empresas y Marketing, Universidad \\ de Sevilla, Calle San Fernando, 4, 41004 Sevilla (e-mail: ppalos@us.es; joseramon.saura@urjc.es) \\ (2) Facultad de Ciencias Jurídicas y Sociales, Dpto. de Economía de la Empresa, Universidad Rey Juan Carlos, \\ Po Artilleros s/n, 28032, Madrid (e-mail: ana.reyes@urjc.es)
}

* Autor a quien debe ser dirigida la correspondencia

Recibido Jul. 18, 2018; Aceptado Sep. 28, 2018; Versión final Ene. 17, 2019, Publicado Jun. 2019

\begin{abstract}
Resumen
El propósito de este trabajo es analizar la literatura sobre el tema de adopción de las tecnologías de computación en la nube (cloud computing). Se pretende proporcionar una visión general de la situación actual de la investigación sobre los servicios en la nube y las principales teorías sobre adopción aplicadas. La metodología utilizada en la investigación es una revisión sistemática de la literatura para la que se han revisado 75 libros, revistas del campo de sistemas de información para identificar y categorizar 24 artículos sobre el tema. El análisis exploratorio de los resultados se ha basado en los contenidos, las metodologías y los modelos de adopción, referenciando los principales trabajos teóricos y empíricos sobre los servicios de computación en la nube. Las conclusiones de esta investigación ponen de manifiesto la importancia de conectar los modelos más utilizados en el campo de la investigación con los trabajos más relevantes realizados hasta la fecha.
\end{abstract}

\section{Models of Adoption of Information Technology and Cloud Computing in Organizations}

\begin{abstract}
The purpose of this paper is to analyze the literature on the subject of adoption of cloud computing technologies. The idea is to provide an overview of the current situation of research on cloud services and major adoption theories. The methodology used in the research is a systematic review of the literature for which 75 books and journals in the field of information systems have been reviewed to identify and categorize 24 articles on the subject. The exploratory analysis of the results has been based on content, methodologies and adoption models, referencing the main theoretical and empirical work on cloud computing services. The conclusions of this investigation show the importance of connecting the most used models in the field of research with the most relevant works carried out to date.
\end{abstract}

Keywords: adoption models; TAM; UTAUT; TOE; cloud computing 


\section{INTRODUCCIÓN}

Para el Instituto Nacional de Estándares y Tecnología de los Estados Unidos de América, el cloud computing (Pearson, 2011; Walterbusch et al., 2013) es un modelo que permite acceder a un conjunto de servicios computacionales (redes, servidores, almacenamiento, aplicaciones y servicios) de manera conveniente cuando se necesita. Esto se debe a que pueden ser rápidamente aprovisionados y liberados con un esfuerzo administrativo y una interacción mínima por parte del proveedor de servicio (Mell y Grance, 2011). Consecuentemente, se toman tecnología, servicios y aplicaciones y se convierten en utilidades que se ponen al servicio de los usuarios cuya adopción posterior resulta crítico para organizaciones y usuarios (Palos y Correia, 2016; Reyes-Menendez et al. 2018a). Los sistemas y el almacenamiento pueden ser utilizados a demanda, evaluando los costes en base a registros de usos anteriores, permitiendo una escalabilidad ágil de los recursos, virtualizando sistemas, reuniendo y compartiendo recursos (Baker, 2011).

El objetivo de este trabajo de investigación es contribuir a un mejor entendimiento de la adopción de esta tecnología y servicio, desde la perspectiva de los modelos e investigaciones publicadas hasta la fecha. Para ello, se realizó una revisión de la literatura que se aborda en el primer apartado. A continuación, se aplicaron estos modelos y se localizaron un conjunto de trabajos que investigaron su adopción por parte de usuarios y organizaciones de diferentes partes del mundo. Finalmente, se aporta un conjunto de conclusiones derivadas de la discusión sobre dichos modelos e investigaciones (Saura et al., 2018a). Las Tecnologías de la Información se han convertido en una parte integral de las organizaciones modernas y desempeñan un papel fundamental en la consecución de ventajas competitivas para la empresa en el entorno competitivo actual (Melville et al., 2004). En este sentido, el Cloud Computing se ha convertido en una importante aplicación de la Tecnología que permite a los usuarios acceder a los recursos de su ordenador sin necesidad de tener esos recursos alojados en el ordenador (Saura et al., 2018b). En su lugar, se encuentran alojados en la nube (Maqueira-Marín et al., 2017; Herráez et al., 2017).

En la Literatura es posible encontrar varios modelos de aceptación de la tecnología que proporcionan marcos útiles para determinar qué factores críticos influyen en la adopción de las TIC por parte del usuario, así como en el uso y comportamiento del mismo dentro de las organizaciones (Ndubisi y Jantan, 2003; Saura et al. 2019). Algunos de los marcos teóricos más importantes son: 1) Teoría de la Acción Razonada (TRA) de Fishbein y Ajzen (1975) y Teoría del Comportamiento Planificado (TPB) de Ajzen (1985); 2) Modelo de Aceptación de la Tecnología (TAM) de Davis (1989); 3) Marco de Tecnología, Organización y Entorno (TOE) de Tornatzky y Fleischer (1990); 4) Teoría de la Difusión de las Innovaciones (DOI) de Rogers (1995); y 5) Teoría unificada de aceptación y uso de la tecnología (UTAUT) (Venkatesh et al., 2003) y UTAUT 2 (Venkatesh et al., 2012).

\section{Teoría de la Acción Razonada (TRA) y Teoría del Comportamiento Planificado (TPB)}

La Teoría de la Acción Razonada (TRA, Theory of Reasoned Action) de Fishbein y Ajzen (1975) ha sido adoptada en muchas disciplinas. Esta teoría explica que las actitudes hacia la realización de una conducta están asociadas positivamente y predicen las intenciones de realizar ese comportamiento. Como puede verse en la Figura 1, TRA propone una serie de constructos interrelacionados (Fishbein y Ajzen, 1975)

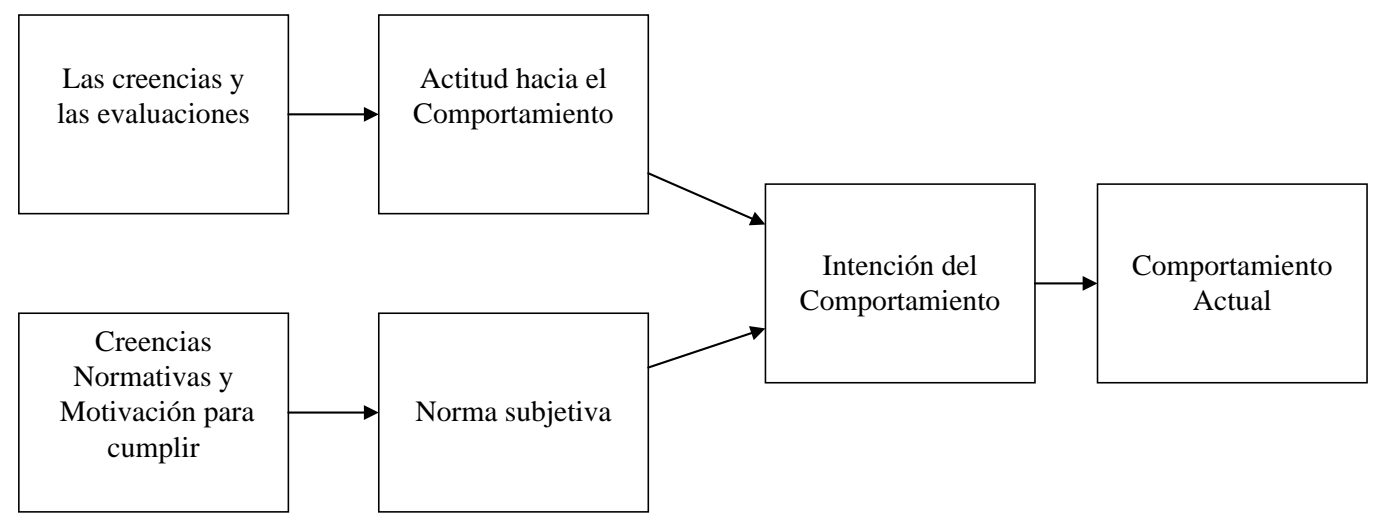

Fig. 1: Teoría de la acción razonada (TRA) (Adaptadad de Fishbein y Ajzen 1975)

Ajzen (1985), desarrolla la Teoría del Comportamiento Planificado (TPB; Theory of Planned Behaviour), añadiendo al modelo TRA dos nuevos constructos con el objetivo de mejorar su capacidad predictiva: Las creencias de control y facilidad percibida y el control de comportamiento percibido (Figura 2). Se trata de una teoría aplicada en numerosos campos de estudio, que van desde el marketing hasta la atención sanitaria. La 
clave de este modelo es la intención de comportamiento. TPB distingue entre tres tipos de creencias: de comportamiento, normativas y de control. Se compone de seis constructos que, de manera colectiva, representan el control real de una persona sobre el comportamiento.

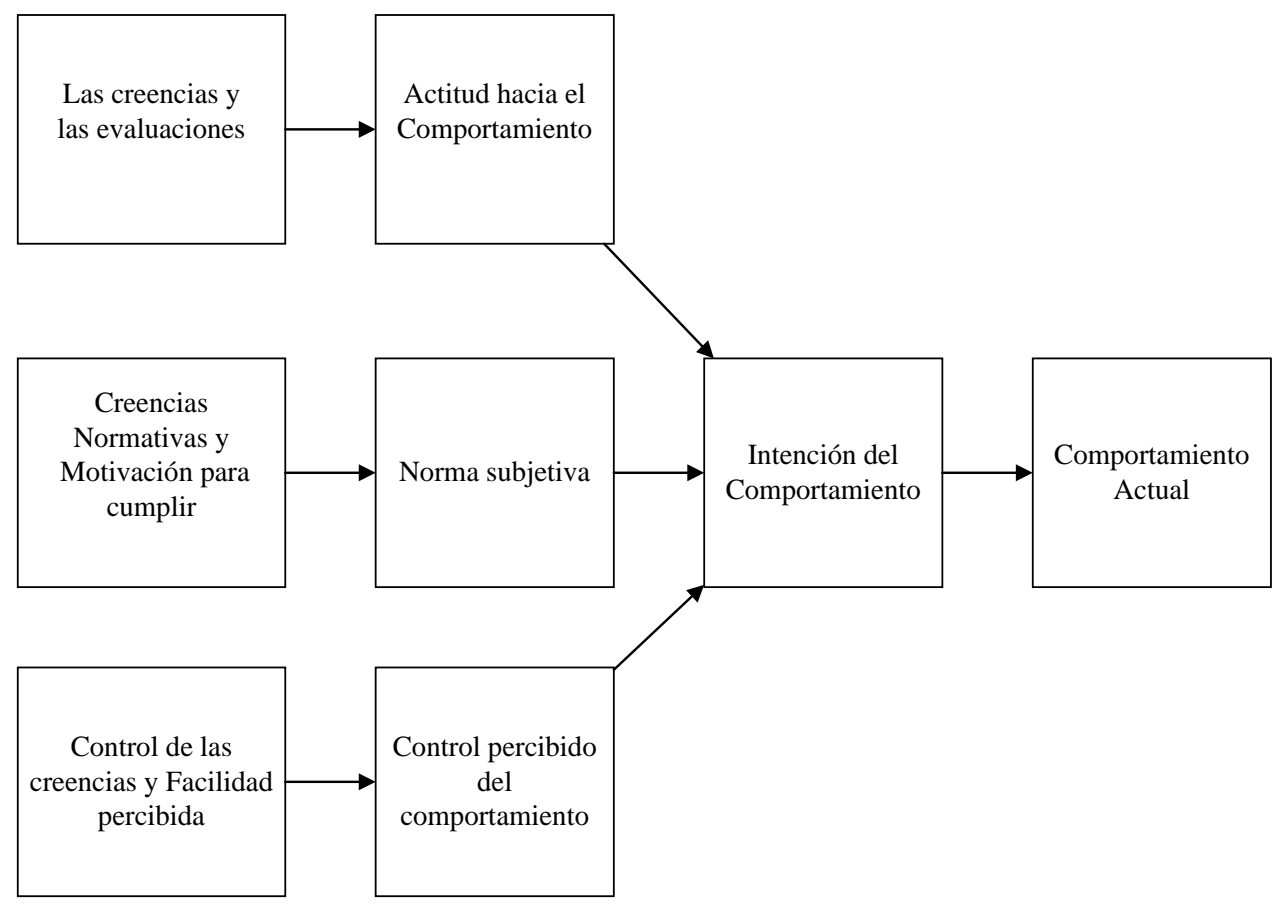

Fig. 2: Teoría del comportamiento planeado (TPB) (Adaptado de Ajzen 1991).

\section{El Modelo TAM}

Con el Modelo de Aceptación de Tecnología (TAM; Technology Acceptance Model; véase Figura 3) de Davis (1989) se busca explicar la relación entre la aceptación y adopción de la tecnología y, posteriormente, la intención de usarla. TAM propone y demuestra empíricamente que la Percepción de Utilidad (PU) y la Percepción de Facilidad de Uso (PFU) son los factores más críticos en el proceso de adopción de la tecnología y el uso de los sistemas (Au y Zafar, 2008; Chen y Tan, 2004). TAM se puede considerar como un caso especial de TRA en el que la utilidad percibida y la facilidad de uso percibida se consideran "creencias y evaluación" (véase Figura 3) que llevan a la actitud, que a su vez conduce a la intención de uso, y finalmente al comportamiento real (Cheung y Vogel, 2013; Joo y Sang, 2013). TAM trata de explicar y predecir el uso de los sistemas de información de los usuarios finales (Davis et al., 1989). Este modelo se ha aplicado en numerosas investigaciones relacionadas con la adopción de tecnología en la organización. TAM explica consistentemente una gran parte de la varianza (40\% según muchos autores, como Legris et al., 2003) en la intención de uso de diversas TIC por parte de usuarios procedentes de numerosos entornos y países (Au y Zafar, 2008; Legris et al., 2003; Hong et al., 2006; Venkatesh y Bala, 2008).

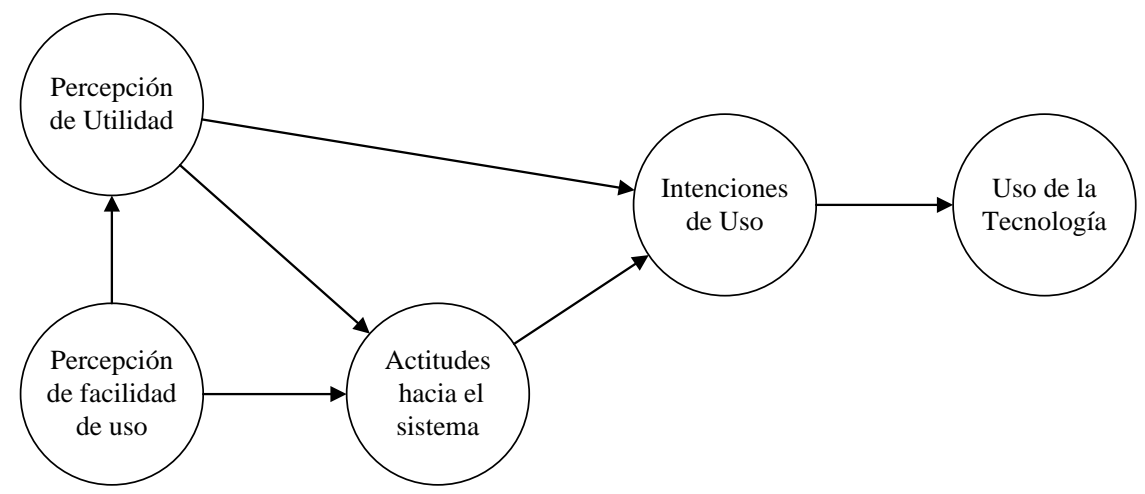

Fig. 3: Modelo TAM original

Algunos de las evoluciones más importantes han sido el TAM 2 de Venkatesh y Davis (2000), la Teoría Unificada de la Aceptación y el Uso de la Tecnología (Unified Theory of Acceptance and Use of Technology, 
UTAUT) de Venkatesh et al. (2003), el modelo de integración de la aceptación de la tecnología y la satisfacción del usuario o el modelo TAM 3, propuesto por Venkatesh y Bala (2008) en el ámbito del comercio electrónico.

\section{El Marco TOE}

El marco TOE (Technology-Organization-Environment; Tornatzky y Fleischer, 1990) propone que la adopción de la innovación tecnológica está influenciada por tres aspectos del contexto de una empresa: el contexto organizacional, relacionado con los recursos y sus características internas, el contexto del entorno, dentro del cual lleva a cabo sus procesos de negocio y el contexto tecnológico, formado por las tecnologías internas y externas relacionadas con la organización que están disponibles en el mercado, sean o no usadas por esta (Baker, 2011). Se trata de un marco para examinar la adopción, a nivel de organizaciones (y no de individuos), de diversos sistemas de información, productos y servicios TIC (ver Figura 4), muy generalizado en la adopción TIC y cuya ventaja es su independencia del tamaño de la empresa (Wen y Chen, 2010), proporcionando una imagen global de la adopción de la tecnología que predice el impacto en las actividades de la cadena de valor y de la difusión posterior de los factores que influyen en las decisiones de negocio (Wang et al, 2010).

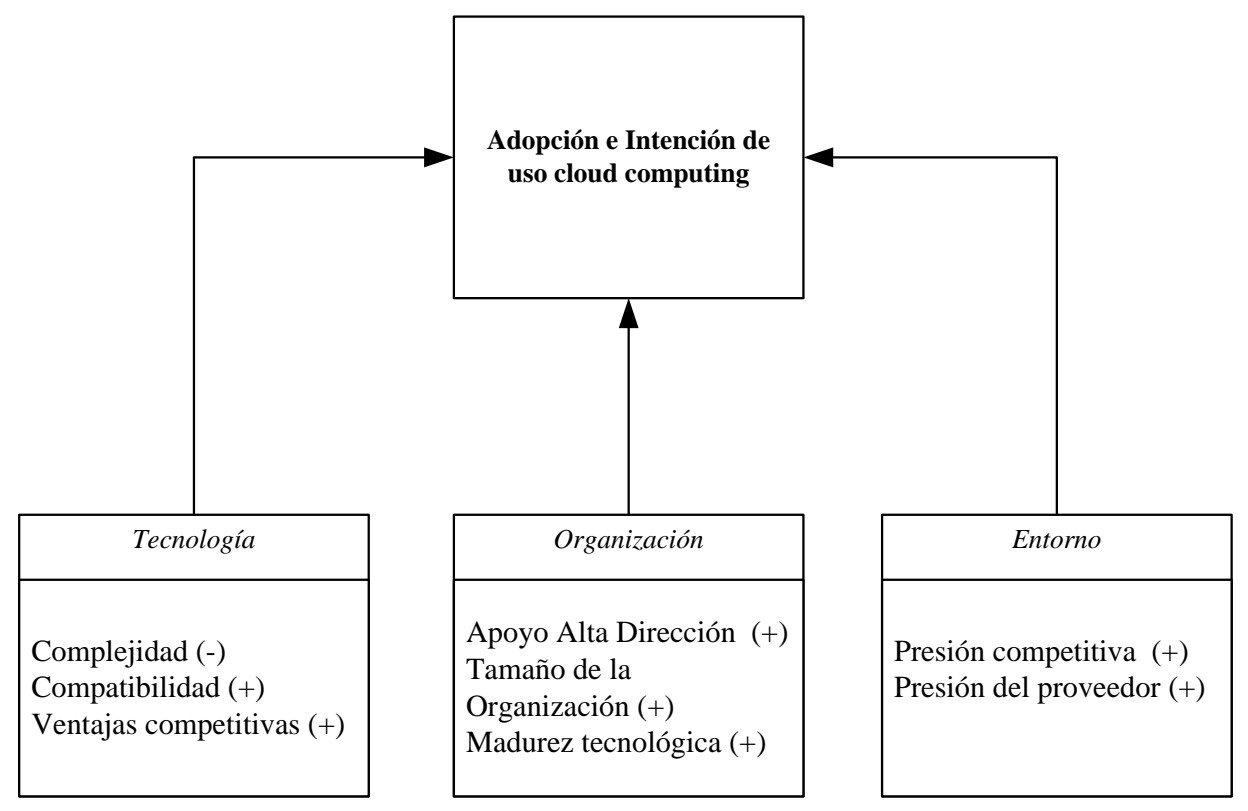

Fig. 4: Modelo TOE (Datos tomados de Low et al. 2011).

\section{Teoría DOI}

Otra teoría de amplia difusión en la literatura sobre aceptación de la tecnología es DOI (Diffusion Of Innovations). Se trata de una teoría, publicada por Rogers en los años 60 y con su última edición en 2003 , que trata de explicar cómo, por qué y en qué medida las nuevas ideas y la tecnología se extienden, operando al nivel de individuos y de empresas. En base a esta teoría, las innovaciones se van comunicando a través de ciertos canales a lo largo del tiempo y dentro de los miembros de un sistema social (Rogers, 1995). Dentro de las empresas, las innovaciones se relacionan con variables independientes tales como las características individuales o de liderazgo, las características internas de la estructura organizativa (centralización, complejidad, formalización, tamaño, etc.) y las características externas a la organización. Dentro del ámbito de los Sistemas de Información, podemos encontrar diversos estudios recientes relacionados con la Teoría DOI (véase, por ejemplo, Li, 2008; Lin y Chen, 2012; Hsu et al., 2014; Low et al., 2011).

\section{Teoría unificada de la aceptación y el uso de la tecnología (UTAUT)}

El modelo UTAUT tiene por objetivo integrar los elementos esenciales de otros modelos y teorías (Venkatesh et al., 2003). Este modelo pretende explicar la aceptación y el uso de la tecnología en las organizaciones. Como se puede ver en la figura 5 , la intención de uso y, a su vez, el uso de la tecnología están determinadas por cuatro variables principales: la expectativa de rendimiento o grado en que una persona considera que el uso del sistema, le ayudará a obtener mejoras de rendimiento en el trabajo; la expectativa de esfuerzo, que se define como la facilidad asociada al uso del sistema; la influencia social o grado en que un individuo percibe que sus referentes sociales creen que debería utilizar el nuevo sistema y las condiciones facilitadoras, o grado en que una persona considera que existe una infraestructura técnica y organizacional para apoyar el uso del sistema. 
El uso de la tecnología se explica directamente por la intención de uso y las condiciones facilitadoras. A su vez, el uso previsto se determina directamente por la expectativa de rendimiento, la expectativa de esfuerzo y la influencia social. Esta teoría también incluye cuatro variables moderadoras de la relación entre las cuatro variables básicas y la intención y el uso de la tecnología: el género, la edad, la voluntad y la experiencia. Venkatesh et al. (2003) informan de que UTAUT explica el 70\% de la varianza de la intención de uso. UTAUT es uno de los modelos más utilizados en los estudios sobre la adopción y el uso de la tecnología.

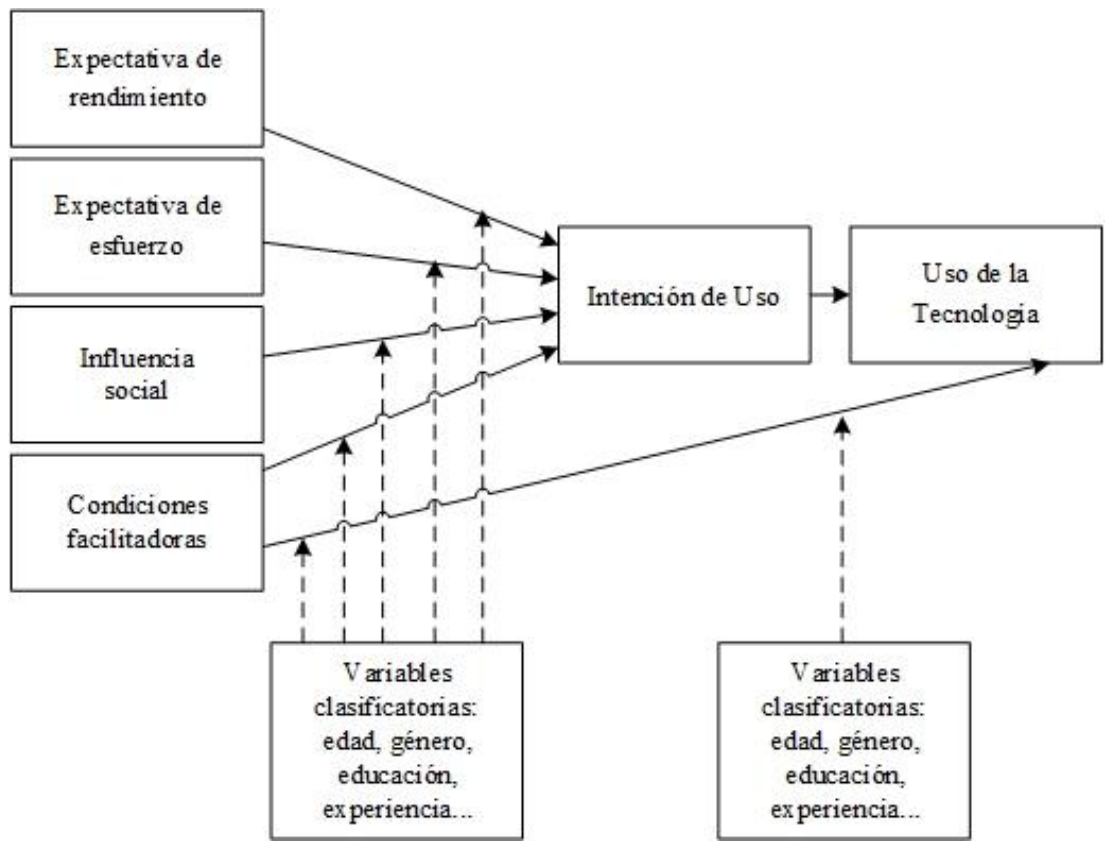

Fig. 5: Teoría Unificada de Aceptación y Uso de Tecnología (UTAUT). (Adaptada de Venkatesh et al. 2003)

Teoría Unificada de la Aceptación y el Uso de la Tecnología 2 (UTAUT 2)

Este modelo, que se muestra en la Figura 6, es una extensión de UTAUT. Tiene como objetivo estudiar la aceptación y el uso de la tecnología en el entorno de consumidores y usuarios. UTAUT2 (Venkatesh et al., 2012) añade al modelo UTAUT original, tres nuevos constructos o bloques de items: la motivación hedonista o la diversión o placer obtenido del uso de la tecnología; el valor del precio o comparación que se hace entre los beneficios que el consumidor percibe y el costo monetario asociado con el uso de la tecnología y el hábito o grado en que una persona tiende a utilizar la tecnología de forma automática, como resultado de un proceso de aprendizaje.

En UTAUT2, el uso de la tecnología está directamente determinado por la intención de uso, las condiciones facilitadoras, como en UTAUT, y, como novedad, por el hábito. A su vez, la intención de uso se explica directamente en la expectativa de rendimiento, la expectativa de esfuerzo y la influencia social, como en UTAUT, y, como novedad, la motivación hedonista y el valor del precio. Las relaciones entre las siete variables (las cuatro originales de UTAUT y las tres exclusivas de UTAUT 2) y la intención de uso y el uso de la tecnología son moderadas por edad, sexo y experiencia. En comparación con el modelo original (UTAUT), el nuevo modelo UTAUT 2 (Venkatesh et al., 2012 produjo una mejora sustancial en la intención de comportamiento de la varianza explicada (56\% a $74 \%$ ) y el uso de la tecnología (de $40 \%$ a $52 \%$ ).

\section{ANÁLISIS DE RESULTADOS}

Se ha llevado a cabo un intenso trabajo de revisión de la literatura, desde la perspectiva de los modelos de adopción expuestos en los apartados anteriores. Anteriores trabajos se centraron en revisiones metodológicas y de la literatura sobre el concepto cloud computing (Walther et al., 2012; Haag y Eckhardt, 2014). Burda y Teuteberg et al. (2014) afirman que, con frecuencia, la literatura tecnológica se centra en abordar cuestiones como la seguridad de la infraestructura cloud mediante la propuesta de nuevas arquitecturas y métodos (véase, por ejemplo, Spillner et al., 2011).

En otros casos, el eje principal de investigaciones son cuestiones relacionadas con oportunidades, costes y riesgos asociados al cloud computing (Benlian y Hess, 2011; Palos y Aguayo-Camacho, 2016), criterios de medición de la calidad del servicio (Benlian et al., 2011) o desafíos relacionados con su adopción, 
disponibilidad del servicio, su rendimiento, la influencia de los proveedores cloud (Maqueira-Marin y BruqueCamara, 2017), la falta de estándares de interoperabilidad y su dificultad de integración y personalización (Géczy et al., 2012; Palos y Correia, 2017). También es posible encontrar varios trabajos que inciden en la importancia de la confianza, tanto en la adopción cloud (Pearson, 2011; Walterbusch et al., 2013), como en las condiciones de privacidad en el almacenamiento de datos (Ion et al., 2011).

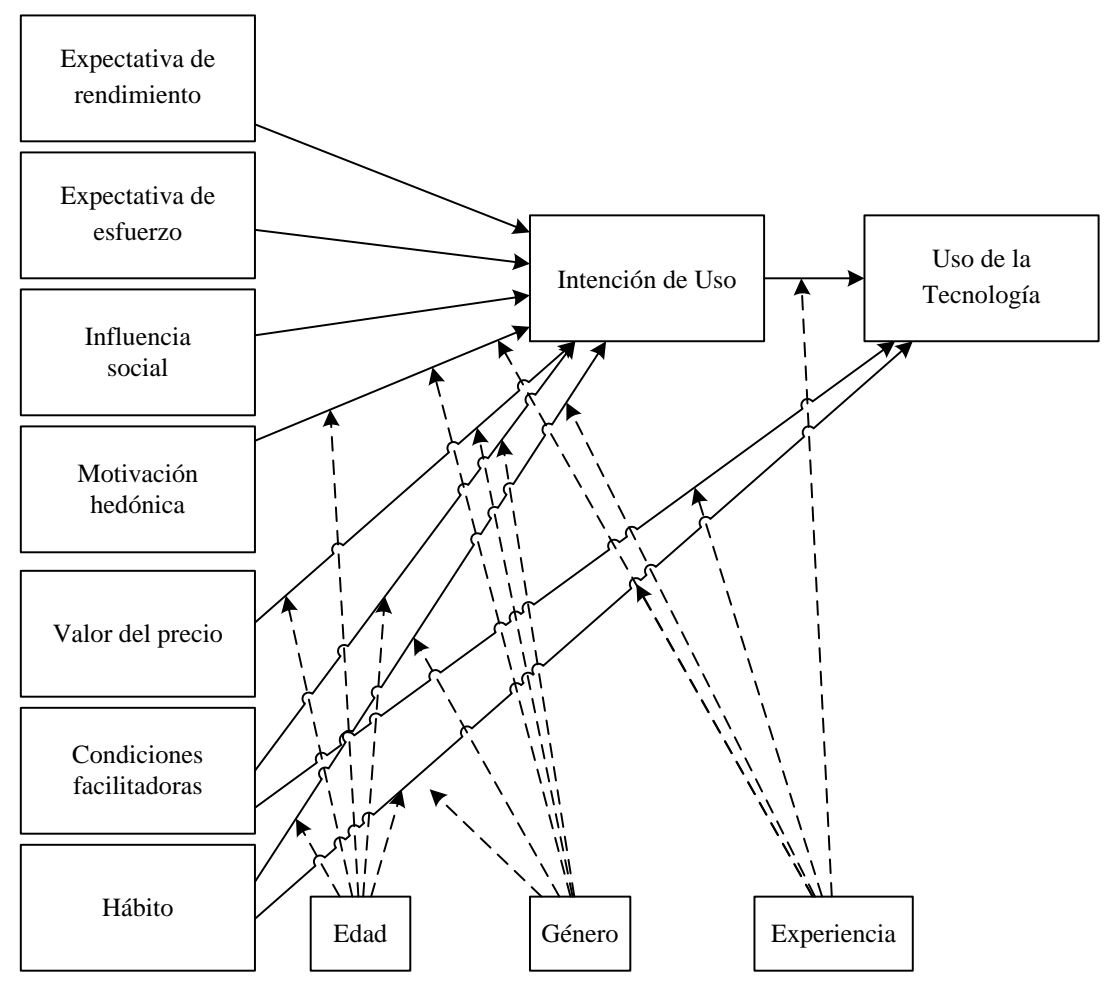

Fig. 6: Teoría Unificada de Aceptación y Uso de la Tecnología 2 (UTAUT 2) (Datos tomados de Venkatesh et al. 2012)

Desde la perspectiva del consumidor final individual es posible encontrar algunos trabajos que analizan la adopción y uso del cloud computing a partir de teorías y modelos mencionados en el apartado anterior, como TPB o TAM (Reyes-Menendez et al. 2018b; Carrillo et al. 2016). Tal es el caso de Bhattacherjee y Park (2014), que estudian la motivación de los usuarios finales para migrar del modelo cliente-servidor al cloud computing, o Giessmann y Stanoevska (2012), que analizan las preferencias del consumidor en un estudio basado en la modalidad PaaS. Wu et al. (2013) proponen un modelo de aceptación que combina TAM con otras variables y es contrastado dentro de una institución universitaria. Moqbel el al. (2014) utilizan también una muestra de estudiantes universitarios para aplicar un marco teórico basado, entre otros, en los modelos TRA y TAM que integra aspectos tales como la compatibilidad, la influencia social y la familiaridad percibida dada la incertidumbre implícita de la nube personal. Una de las más importantes conclusiones, está en que los usuarios utilizan la nube por su compatibilidad con su estilo de vida y trabajo diario, hecho que aumenta conforme se van familiarizando más con ella (Guerrero et al. 2016).

Por lo que respecta a investigaciones sobre la adopción de los sistemas cloud en organizaciones, las investigaciones concluyen que la relevancia de aspectos tales como la incertidumbre, la compatibilidad, el apoyo de la alta dirección, la utilidad percibida, la facilidad de uso de la tecnología, la experiencia previa, las restricciones geográficas, el tamaño de la empresa, el mercado, los esfuerzos de los proveedores, la seguridad, la confianza, la influencia social y la presión de socios comerciales en la adopción de cloud computing (Gangwar et al., 2015; Lin y Chen, 2012; Low et al, 2011; Wu, 2011). Los trabajos que han resultado más significativos en nuestra búsqueda de variables explicativas, en la aplicación de TOE, cuentan con las variaciones propias de un marco abierto y cierta disparidad en la metodología, la elección de variables y en los resultados. Tal es el caso de Alshamaila et al (2012), Abdollahzadehgan et al. (2013), Nkhoma y Dang (2013) y Yang et al. (2015). Nkhoma y Dang (2013) emplean datos secundarios de una encuesta a una gran empresa de servicios (IBM), para determinar elementos que impulsan u obstaculizan la adopción del cloud computing; en el ámbito de las Pymes (Pequeñas y Medianas Empresas), Alshamaila et al. (2012) intentan identificar los principales factores para la adopción de servicios en la nube, a través de un estudio exploratorio en empresas del noreste de Inglaterra, resultando que la ventaja relativa, la incertidumbre, las restricciones geográficas, la compatibilidad, la capacidad de prueba, el tamaño de la empresa, el apoyo de la alta dirección, las experiencias previas, la innovación, la industria, el alcance del mercado, los esfuerzos de los proveedores 
y el apoyo informático externo son determinantes; Yang et al. (2015), que proponen un modelo para analizar la adopción de innovaciones tecnológicas basadas en SaaS, en una muestra de 173 empresas chinas, encontrando que el apoyo de la alta dirección, la ventaja relativa, la simplicidad, la compatibilidad o la presión de competidores y socios resultaron significativos.

Otro grupo de estudios proponen distintos modelos de adopción cloud usando la Teoría DOI (Lin y Chen, 2012), que realizan una entrevista semiestructurada entre 19 profesionales TIC de Taiwán, demostrando que las empresas rechazan adoptar la nube hasta que las incertidumbres asociadas con la tecnología cloud, como la seguridad o la estandarización, se reducen, posibilitando así nuevas ventajas en su modelo de negocio. Otros estudios integran teorías DOI y TOE (Low et al., 2011; Oliveira et al., 2014). Hsu et al. (2014) combinan aspectos de DOI y TOE en la adopción del cloud en la industria de alta tecnología de Taiwán, con una muestra de 200 empresa, concluyendo que la adopción del cloud está todavía en su etapa inicial y que los beneficios esperados, los riesgos y problemas percibidos, los recursos y el personal TIC son los principales determinantes. Oliveira et al. (2014) proponen un modelo similar, pero más completo, en una muestra de 369 empresas portuguesas, considerando que la innovación es concebida como un proceso de comunicación que utiliza los diferentes canales dentro del sistema social. Distinguen tres tipos de factores de adopción: factores individuales (el liderazgo, la actitud hacia el cambio), la estructura organizativa interna (la centralización, la complejidad, la interconexión, el número de empleados y la holgura de la organización), y las características externas (apertura del sistema) de la organización, demostrando que la ventaja relativa, la complejidad, la preparación tecnológica, el apoyo de la alta dirección y el tamaño de la empresa tienen un efecto directo en la adopción de los sistemas cloud.

\section{DISCUSIÓN}

Gangwar et al. (2015), destacan la importancia de abordar el impacto de aspectos específicos de la empresa, empleando para ello una muestra de 280 empresas indias.

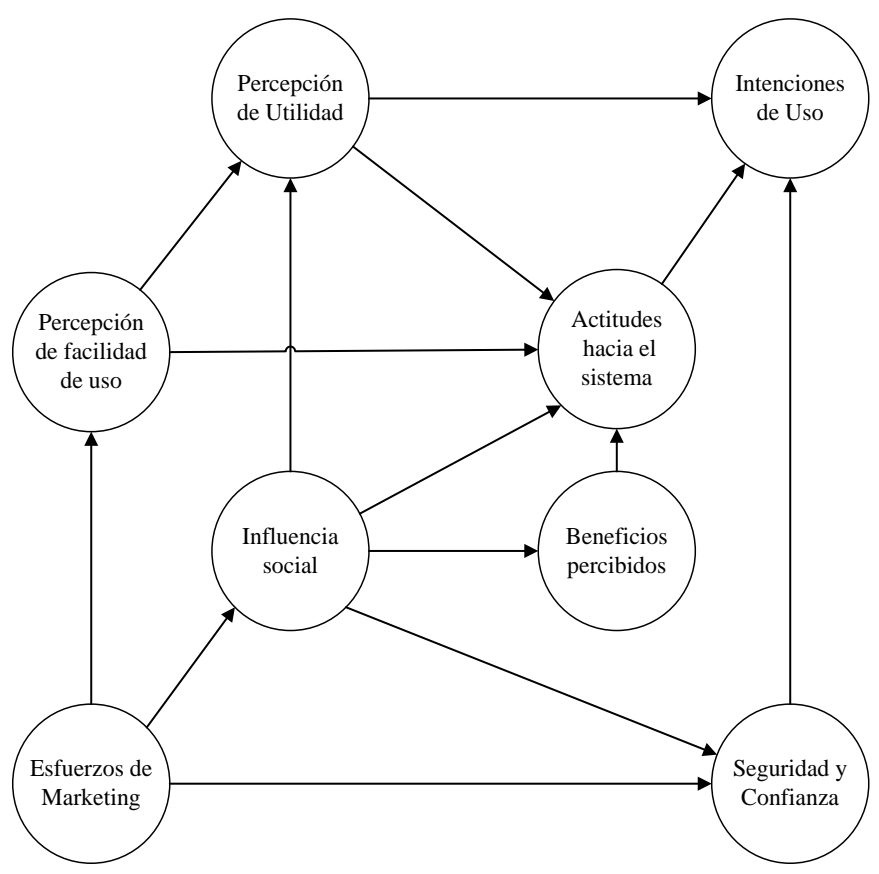

Fig. 7: Modelo mejorado (Datos tomados de Wu 2011)

Desarrollaron un modelo TAM-TOE, que combina, las variables habituales de TAM con variables identificadas en diversos estudios y relacionadas con los tres aspectos del contexto de una empresa que se incluyen en el marco TOE, concluyendo que todas las variables de los tres contextos son determinantes importantes para la adopción del cloud computing, ya sea de manera directa o indirecta. Sin embargo, Wu (2011) encontró algunas limitaciones en el modelo, sintiendo la necesidad de explorar algunos determinantes cruciales para la adopción de servicios de cloud computing: la seguridad y la confianza, los beneficios percibidos y los esfuerzos de marketing. El modelo propuesto por Wu (2011), que se representa en la figura 7, se basa en el original de TAM y también en el modelo TAM-DTM, el modelo de seguridad y la confianza S\&T (Shin, 2009) y el estudio de Lin y Chen (2012), que incluyó el constructo Esfuerzos de Marketing, entendiendo por tal los esfuerzos para comercializar los nuevos servicios cloud que la organización ofrecerá tras la adopción. A su vez, pueden desempeñar un papel importante en la etapa inicial de adopción de estos servicios y mejorar su intención de uso (Fortes et al., 2016). 


\section{CONCLUSIONES}

La adopción de servicios Cloud Computing empieza a ser un objeto de estúdio cada vez más común en los trabajos de investigación sobre gestión de sistemas de información. Este trabajo ha pretendido conectar los modelos más utilizados en el campo de la investigación con los trabajos más relevantes hasta ahora realizados. Se concluye que existen dos tipos de trabajos, fundamentalmente, aquellos cuya muestra son organizaciones y otros, donde lo son los usuarios del sistema cloud. En ambos casos, las conlusiones son relevantes, pero tal vez revistan de más interés aquellos sobre organizaciones, que aún son escasos. En cualquier caso, en muchos trabajos el principal reto es predecir y fundamentar cómo se adopta esta tecnología a través de la superación de las barreras que afectan, por ejemplo, a su percepción de utilidad o a la intención de uso. Como consideración final, son los modelos TAM ampliados con variables externas y el marco TOE, combinado, en algunos estudios, con $\mathrm{DOI}$, son los que más citas han obtenido en la Literatura revisada. Ahora bien, parece que existe una carencia de trabajos en factores como el tiempo de adopción y despliegue de la nube, la cooperación empresarial que evaluaría el efecto que tendría la participación en redes empresariales destinadas a aumentar la capacitación organizativa y el reciclaje del personal técnico en la adopción de los sistemas cloud, la estandarización de interfaces y plataformas tecnológicas

\section{REFERENCIAS}

Ajzen, I., From intentions to actions: A theory of planned behavior. En Kuhl J. y J. Beckmann, Action control: From cognition to behavior, 11-39, Berlin, Heidelber, New York, Springer-Verlag (1985)

Ajzen, I., The theory of planned behavior, Organizational Behavior and Human Decision Processes, 50 (2), $179-211$ (1991)

Alshamaila, Y., S. Paiannidis y F. Li., Cloud computing adoption by SMEs in the north east of England. A multi-perspective framework, Journal of Enterprise Information Management, 26(3), 250-275 (2012)

Au, Y. y H. Zafar, A multi-country assessment of mobile payment adoption, Working Paper Series No. 0055IS-296-2008. College of Business, University of Texas, San Antonio, TX (2008)

Baker, J., The technology-organization-environment framework. En Dwivedi, Y., M. Wade y S. Schneberger (Eds.), Information Systems Theory: Explaining and Predicting Our Digital Society, Springer, New York, NY, 231-246 (2011)

Benlian, A., M. Koufaris y T. Hess, Service quality in software-as-a-service: Developing the SaaS-qual measure and examining its role in usage continuance, Journal of Management Information Systems, 28(3), 85-126 (2011)

Benlian, A., T. Hess y P. Buxmann, Drivers of saas-adoption an empirical study of different application types, Business Information Systems Engineering, 1, 357-369 (2009)

Bhattacherjee, A. y S. Park, Why end-users move to the cloud: A migration-theoretic analysis, European Journal of Information Systems, 23(3), 357-372 (2014)

Burda, D. y F. Teuteberg, The role of trust and risk perceptions in cloud archiving, Journal of High Technology Management Research, 25(2), 172-187 (2014)

Carrillo, M.H. C. Franky y otros dos autores, SCLOUDPY: Sistema Informático Web de Multi-Tenencia para el Procesamiento en la Nube de Pedidos de PYMES, Inf. Tecnol., 27 (1), 181-194 (2016)

Cheung, R. y D. Vogel, Predicting user acceptance of collaborative technologies: An extension of the technology acceptance model for e-learning, Computers \& Education, 63, 160-175 (2013)

Davis, F.D., Perceived usefulness, perceived ease of use, and user acceptance of Information Technology. MIS Quartely 13(3), 319- 340 (1989)

Fishbein, M. e I. Ajzen, Belief, Attitud Intention, and Behavior: An Introduction to Theory and Research. Reading, MA: Addison-Wesley (1975)

Fortes, N., J.H. Pereira y J.F.D. Costa, A adoção de serviços cloud computing pelas empresas portuguesas: O papel dos esforços de marketing, RISTI-Revista lbérica de Sistemas e Tecnologias de Informação, (18), 33-48 (2016)

Gangwar, H., H. Date y R. Ramaswamy, Understanding determinants of cloud computing adoption using an integrated TAM-TOE model, Information Technology, Journal of Entreprise Information, 28 (1), 107-130 (2015)

Géczy, P., N. Izumi y H. Kôiti, Cloudsourcing: managing cloud adoption, Global Journal of Business Research, 6 (2), 57 70 (2012)

Giessmann, A. y K. Stanoevska, Platform as a service, A conjoint study on consumers preferences, International Conference on Information Systems, Orlando, FL, US (2012)

Guerrero, A., Carlos y J. Londoño, Revisión de la Problemática de la Calidad del Software para el Desarrollo de Aplicaciones de Computación en la Nube, Inf. Tecnol., 27 (3), 61-80 (2016)

Haag, S. y A. Eckhardt, Organizational cloud service adoption: a scientometric and content-based literature analysis, Journal of Business Economics, 84(3), 407-440 (2014) 
Herráez, B., D. Bustamante y J.R. Saura, Information classification on social networks, Content analysis of e-commerce companies on Twitter, Revista Espacios, 38(52), 16, (2017)

Hong, S.-J., J. Thong y K. Y. Tam, Understanding continued information technology usage behavior: a comparison of three models in the context of mobile internet, Decision Support Systems, 42 (3), 1819-1834 (2006)

Hsu, P-F., R. Soumya, Y-Y. Li-Hsieh, Examining cloud computing adoption intention, pricing mechanism and deployment, International Journal of Information Management, 34, 474-488 (2014)

Ion, I., N. Sachdeva, P. Kumaraguru y S. Capkun, Home is safer than the cloud: Privacy concerns for consumer cloud storage, $7^{\text {th }}$ Symposium on Usable Privacy and Security, Pittsburgh, Pennsylvania (2011)

Joo, J. e Y. Sang, Exploring Koreans' smartphone usage: An integrated model of the technology acceptance model and uses and gratifications theory, Computers in Human Behavior, 29(6), 2512-2518 (2013)

Legris, P., J. Ingham y P. Collerette, Why do people use information technology? A critical review of the technology acceptance model, Information and Management, 40 (3), 191-204 (2003)

Lin, A. y N. Chen, Cloud computing as an innovation: Percepetion, attitude, and adoption, International Journal of Information Management, 32 (6), 533-540 (2012)

Low, C., Y. Chen y M. Wu, Understanding the determinants of cloud computing adoption, Industrial Management \& Data Systems, 111(7), 1006-1023 (2011)

Maqueira-Marín, J.M., S. Bruque-Cámara y B. Minguel-Rata, Environment determinants in business adoption of Cloud Computing, Industrial Management \& Data Systems, 117 (1), 228-246 (2017)

Mell, P. y T. Grance, Definition of cloud computing, NIST (2011)

Melville, N., K. Kraemer y V. Gurbaxani, Review: information technology and organizational performance: An integrative model of IT business value, MIS Quarterly, 28 (2), 283-322 (2004)

Moqbel, M., V. Bartelt y M. Al-Suqri, A Study of Personal Cloud Computing: Compatibility, Social Influence, and Moderating Role of Perceived Familiarity, Twentieth Americas Conference on Information Systems, Savannah (2014)

Nkhoma, M. y D. Dang, Contributing factors of cloud computing adoption: a technology-organisation-environment framework a roach, International Journal Information System Engineering, 1, 38-49 (2013)

Oliveira, T., M. Thomas, M. Espadanal, Assessing the determinants of cloud computing adoption: An analysis of the manufacturing and services sectors, Information and Management, 51, 497-510 (2014)

Palos, P.R. y M.B. Correia, La actitud de los recursos humanos de las organizaciones ante la complejidad de las aplicaciones SaaS. Dos Algarves: A Multidisciplinary e-Journal, 87-103 (2016)

Palos, P.R. y M.B. Correia, The Paradigm of the Cloud and Web Accessibility and its Consequences in Europe, 7th International Conference on Software Development and Technologies for Enhancing Accessibility and Fighting Infoexclusion (DSAI 2016). ACM. Vila Real, Portugal (2017)

Palos, P.R. y M. Aguayo-Camacho, Los cambios organizacionales y la nube en el entorno turístico: estudio de caso, International Journal of World of Tourism, 3 (5) 33-42 (2016)

Pearson, S., Toward accountability in the cloud, IEEE Computer Society, 15(4), 64-69 (2011)

Reyes-Menendez, A., J. Saura, P. Palos-Sanchez y J. Alvarez-Garcia, Understanding User Behavioral Intention to Adopt a Search Engine that Promotes Sustainable Water Management, Symmetry, 10(11), 584 (2018b)

Reyes-Menendez, A., P.R. Palos-Sanchez, J.R. Saura y F. Martin-Velicia, Understanding the Influence of Wireless Communications and Wi-Fi Access on Customer Loyalty: A Behavioral Model System, Wireless Communications and Mobile Computing, 2018, 1-16 (2018a)

Rogers, E.M., Diffusion of Innovations (4rd Ed.). New York: 4th ed. Free Press (1995)

Saura, J.R., P.R., Palos-Sanchez y M. B Correia, Digital Marketing Strategies Based on the E-Business Model: Literature Review and Future Directions. In Organizational Transformation and Managing Innovation in the Fourth Industrial Revolution, 86-103, IGI Global (2019)

Saura, J. R., P.R. Palos-Sanchez y M. A. Rios Martin, Attitudes to environmental factors in the tourism sector expressed in online comments: An exploratory study. International Journal of Environmental Research and Public Health, 15(3), 553. (2018a)

Saura, J.R., A. Reyes-Menendez y C. Alvarez-Alonso, Do Online Comments Affect Environmental Management? Identifying Factors Related to Environmental Management and Sustainability of Hotels, Sustainability, 10(9), 3016 (2018b)

Shin, D. H., Towards an understanding of the consumer acceptance of mobile wallet, Computers in Human Behavior, 25(6), 1343-1354 (2009)

Spillner, J., G. Bombach, S. Matthischke, J. Muller, R. Tzschichholz y A. Schill, Information dispersion over redundant arrays of optimal cloud storage for desktop top users, 4th IEEE International Conference on Utility and Cloud Computing (UCC), Melbourne, Australia (2011)

Tornatzky, L. y M. Fleischer. The Process of Technology Innovation. Lexington, MA, Lexington Books (1990) 
Venkatesh, V. y F.D. Davis, A Theoretical Extension of the Technology Acceptance Model: Four Longitudinal Field Studies, Management Science, 46, 2, 186-204 (2000)

Venkatesh, V., J. Thong y X. Xu, Consumer Acceptance and Use of Information Technology: Extending the Unified Theory of Acceptance and Use of Technology. MIS Quarterly (36:1), 157-178 (2012)

Venkatesh, V., M. Morris, G.B. Davis y F.D. Davis, User acceptance of information technology: Toward a unified view. MIS Quarterly, 27(3), 425-478 (2003)

Venkatesh, V. y H. Bala, Technology acceptance model 3 and a research agenda on interventions. Decision Sciences, 39(2), 273-315 (2008)

Walterbusch, M., B. Martens y F. Teuteberg, Evaluating cloud computing services from a total cost of ownership perspective, Managent Research Review, 36, 613-638 (2013)

Walther, S., A. Plank, T. Eymann, N. Singh y G. Phadke, Success factors and value propositions of software as a service provider: a literature review and classification. In: Proceedings of the 18th Americas conference on information systems, Seattle (2012)

Wang, Y.M., Y.S. Wang y Y.F. Yang, Understanding the determinants of RFID adoption in the manufacturing industry, Technological Forecasting \& Social Change, 77, 803-815 (2010)

Wen, K. W. e Y. Chen, E-business value creation in Small and Medium Enterprises: a US study using the TOE framework, International Journal of Electronic Business, 8(1), 80-100 (2010)

Wu, W.W., Developing an explorative model for SaaS adoption, Expert systems with applications, 38(12), 1505715064 (2011)

Wu, W.-W. y L. W.-T. Lan, Factors Hindering Acceptance of Using Cloud Services in University: A Case Study, The Electronic Library, 31(1), 84-98 (2013)

Yang, Z., J. Sun, Y. Zhang e Y. Wangd, Understanding SaaS adoption from the perspective of organizational users: A tripod readiness model, Computers in Human Behavior 45, 254-264 (2015) 